\title{
La competencia china para las exportaciones de manufactura mexicana hacia Estados Unidos en las dos últimas décadas
}

A ctualmente México y China son considerados dos importantes exportadores de manufactura en el comercio mundial. El sector manufacturero representa en ambos países el principal sector de exportación, y es por esta razón que las exportaciones de manufactura son una indispensable entrada de recursos e inversión que contribuye al desarrollo de los principales sectores de la economía.

En este documento se presenta cómo México y China han competido con sus exportaciones de manufactura en el mercado estadounidense, tomando en consideración que Estados Unidos es el principal importador de manufactura a nivel mundial.

\section{La importancia del sector manufacturero mexicano}

Lograr una apertura de la economía y poder dirigir la dinámica comercial hacia el exterior fueron dos de los principales objetivos perseguidos con las reformas macroeconómicas llevadas a cabo en México a partir de 1982 para sanear a la economía interna después de la manifestación de la crisis.

Así, el proceso de liberalización y de apertura de la economía se encaminó a modificar el patrón de industrialización,-que por décadas descansó en el modelo de sustitución de importaciones-en la perspectiva de alcanzar un mayor nivel de productividad y competitividad para dirigir la dinámica de desarrollo en torno al mercado internacional. Para lograr esta meta se fomenta el desarrollo y la especialización de

* Pasante de la maestría en ciencias sociales, CUCSH, Universidad de Guadalajara. los principales sectores de la economía; en la industria, los productos intensivos en mano de obra se convierten en el "arma principal" con la que nuestro país se inserta en el mercado mundial -desplazando a los hidrocarburos, sector bajo el cual se había sustentado el intercambio con el exterior por más de cuatro décadas- Desde la mitad de la década de 1980 y hasta la actualidad, el de manufactura es el principal sector de exportación como se muestra en el cuadro 1.

En el cuadro 1 observamos que en 1983 las exportaciones de manufactura ocupaban 24.44 por ciento de las exportaciones totales de mercancías, mientras que en el 2000 ocuparon 55.50 por ciento del total - sin incluir la maquila -. En 1983 las exportaciones no petroleras pasaron de 5 mil 452 millones de dólares, a 23 mil 54 millones de dólares en el 2000. ${ }^{1}$ Así mismo, entre las exportaciones de manufacturas, pierden participación relativa las tradicionales y aumentan las manufacturas dinámicas -por ejemplo las del sector automotriz- que son monopolizadas en la actualidad por las empresas multinacionales.

La estrategia de promoción de exportaciones, junto a la estrategia de crecimiento de las corporaciones trasnacionales establecidas en el país, han llevado a que el crecimiento de las exportaciones manufactureras se realice hacia el mercado de EUA, dado su tamaño y cercanía, además que este país es el principal acreedor de manufactura a nivel mundial. De acuerdo a lo anterior y como se muestra en el cuadro 1 , el cambio en la composición de las exportaciones de la economía mexicana ha reflejado una participación creciente de las manufacturas junto con una reducción relativa de los hidrocarburos lo que, 
Cuadro 1

Composición de las exportaciones mexicanas después de la apertura comercial (años seleccionados, porcentajes)

\begin{tabular}{|c|c|c|c|c|c|c|c|}
\hline Sector & 1983 & 1986 & 1989 & 1992 & 1995 & 1998 & $2000^{*}$ \\
\hline Agropecuarias & 5.33 & 13.36 & 7.07 & 8.92 & 8.08 & 7.92 & 8.54 \\
\hline Extractivas & 70.23 & 36.63 & 34.69 & 36.27 & 32.62 & 34.29 & 35.96 \\
\hline Manufacturas ${ }^{* *}$ & 24.44 & 48.01 & 57.61 & 54.81 & 59.3 & 57.79 & 55.5 \\
\hline Total & 100 & 100 & 100 & 100 & 100 & 100 & 100 \\
\hline
\end{tabular}

*estimado.

**Las exportaciones de manufactura no incluyen la maquila.

Fuente: Elaboración propia, con base en indicadores de comercio exterior del Banco de México.

entre otras cosas, ha reforzado el comercio bilateral con el mercado estadounidense. Este mercado ha pasado a ser motor de crecimiento para las industrias con capacidad competitiva y de reestructuración para hacer frente al contexto de economía abierta. En 1981, 32 por ciento de las exportaciones hacia Estados Unidos eran de productos manufacturados; en el 2000, dicho porcentaje fue de 69 por ciento. ${ }^{2}$

Esta mayor integración ha obedecido también al proceso de internacionalización que se ha acentuado en los últimos años, en el cual las empresas trasnacionales buscan ventajas comparativas qué explotar y desarrollar para reducir costos y mejorar su posición competitiva en el mercado internacional. Es ello lo que ha acentuado el comercio bilateral de manufacturas entre México y Estados Unidos, esto a nivel intraindustrial principalmente.

Como se mencionó al iniciar este documento, aquí se pretende dar cuenta de un potencial competidor internacional, al sector de manufactura mexicano: China. Dado el grado de desarrollo alcanzado por esta nación en las dos ultimas décadas, $y$ habiéndose colocado como uno de los principales competidores en el mercado mundial de bienes de capital a través de las exportaciones en productos intensivos en mano de obra, surge fácilmente la pregunta: ¿hasta qué grado la posición de China en el escenario

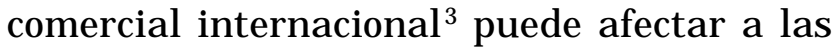

exportaciones de manufactura mexicana principalmente en el mercado estadounidense?

\section{El papel del sector manufacturero en el desarrollo económico de China}

En China con el modelo de "puertas abiertas" de finales de los años setenta, el gobierno dirigido por el Partido comunista- lleva a cabo una serie de reformas macroeconómicas que conducen a la apertura de la economía y rompen con un modelo de tradición milenaria que ignoraba -o digamos, dejaba en un segundo plano- el comercio con el exterior.

Los resultados de las reformas han sido por demás exitosos; la importancia de este país como competidor internacional radica en que en los últimos veinte años la economía interna de China ha registrado un crecimiento anual promedio de 9.8 por ciento, lo cual coloca a esta nación en el séptimo lugar en términos de fuerza económica a escala mundial aunque China es todavía un país en desarrollo. ${ }^{4}$

es necesario conocer cómo se comportan las manufacturas chinas en el mercado mundial, sobre todo las exportaciones de manufactura hacia el mercado estadounidense
Desde la apertura comercial, China ha sido considerado un país que inunda el mercado internacional con productos intensivos en mano de obra y de bajo costo. Es precisamente el sector de la manufactura el que convierte a esta nación en uno de los más importantes competidores comerciales en el ámbito. Desde la mitad de la década de1980, 
Cuadro 2

\begin{tabular}{cc}
$\begin{array}{c}\text { Evolución de las exportaciones de manufacturas de China desde la apertura comercial } \\
\text { (porcentaje respecto al total de exportaciones, años seleccionados) }\end{array}$ \\
\hline Año & Porcentaje sobre exportaciones totales \\
1970 & 28.9 \\
1975 & 35.6 \\
1980 & 49.2 \\
1984 & 54.3 \\
1985 & 49.5 \\
1988 & 69.7 \\
1990 & 74.4 \\
1992 & 79.9 \\
1993 & 78.7 \\
1995 & 81.2 \\
1996 & 81.9 \\
1998 & 83.1 \\
$2000^{*}$ & 85.4 \\
\hline
\end{tabular}

${ }^{\star}$ Estimado

Fuente: Estadísticas del International Trade Center

el sector de manufactura ocupa un lugar prioritario para el desarrollo de su comercio exterior.

Como se puede apreciar en el cuadro 2 las exportaciones de manufacturas desde 1978, año en que entra en vigor el modelo de "puertas abiertas", han ocupado un porcentaje importante del total de exportaciones. A partir de 1990, las exportaciones de manufactura representan más de 50 por ciento de las exportaciones totales.

La importancia del mercado estadounidense en el desarrollo del sector manufacturero de México y China

Considerando que Estados Unidos es el principal importador de manufactura a nivel mundial, ${ }^{5}$ que es el principal destino de las exportaciones de México y además su primer socio comercial, las relaciones político-comerciales de estos dos países en los últimos veinte años, tienen una importancia crucial para entender el desarrollo $y$ el alcance competitivo del sector manufacturero mexicano en el ámbito internacional. Por ejemplo, ser miembro del TLCAN, así como pertenecer al mercado norteamericano le ha permitido a México superar a China en el volumen de exportación manufacturera hacia el mercado estadounidense. En el cuadro 3 se aprecia este hecho.

Así mismo, el acceso de China al comercio mundial en las últimas dos décadas ha estado regido en cierta forma por las relaciones políticocomerciales con Estados Unidos -que a diferencia de México han sido más restrictivasen parte por la difícil relación política que data de la Posguerra. ${ }^{6}$

Después de más de tres décadas de fricciones comerciales entre Estados Unidos y China, -es decir, después del otorgamiento y las sucesivas renovaciones del status de Nación más favorecida $\mathrm{NMF}^{7}$ por parte de EUA a Chinaen 1999 se firma un Acuerdo Bilateral para postular a China como miembro de la OMC, lo que se logra en diciembre del 2001. Esto implicará para China gozar de inmediato de los beneficios y obligaciones como país miembro y subdesarrollado.

Desde la última renovación del Status de NMF a China en $1997^{\circ}$ y el Acuerdo Bilateral Estados Unidos-China para la postulación de este último a la OMC firmado en 1999, se presupone una nueva era de liberalización y 
cooperación en las relaciones comerciales entre ambos países, lo que podría cambiar la ventaja que ha tenido México sobre China en volumen de exportaciones de manufacturas.

\section{El Tratado de Libre Comercio de América del Norte: ventaja para México}

Antes y después de la entrada en vigor del TLCAN, las exportaciones manufactureras chinas -y en general las asiáticas-hacia Estados Unidos, se vieron superadas por las mexicanas, ${ }^{9}$ como se muestra en el cuadro 3.

El primer aspecto que salta a la vista en el cuadro 3 es que dentro de los países que integran el TLCAN el principal proveedor de manufacturas para Estados Unidos es Canadá. Cabe notar que a pesar de que las exportaciones de manufactura de este país hacia Estados Unidos se vieron reducidas en el período 19861988, después de los años que anteceden a la firma del tratado, se recuperan de nuevo las compras de manufactura procedentes de Canadá.

Como se muestra en las estadísticas, Estados Unidos realizó una mayor compra de manufactura procedente de América del Norte que de China en el período 1985-2000. En el mismo período, de las importaciones totales de manufactura, las procedentes de México aumentaron de 3.77 por ciento a 14.99 por ciento; mientras que las importaciones de manufactura procedentes de China aumentaron de 1.17 a 4.13 por ciento.
Ahora bien hay que destacar que desde la entrada en vigor del TLCAN en 1994 se favoreció la compra de manufacturas por parte de Estados Unidos a los miembros del tratado, lo que desvió una mayor penetración de las manufacturas chinas hacia Estados Unidos. Desde el año en que entra en vigor el tratado, hasta la actualidad, las exportaciones de manufactura mexicana hacia Estados Unidos, han ido en aumento considerable, mientras que se presenta un estancamiento de las importaciones de manufacturas chinas por parte de éste. Incluso, desde 1998, las cifras son aún más bajas que en $1992-4.96$ por ciento- lo que significa un retroceso.

Sin embargo, es importante reconocer que las cifras respecto a la manufactura china no han bajado de 4 por ciento desde 1992; podría suponerse que las relaciones comerciales entre ambos países desde ese año se encuentran en un proceso de ajuste y afianzamiento que los prepara para el nuevo contexto económico que se presenta con la entrada de China a la Organización Mundial de Comercio, sin duda este elemento se traduce en un nuevo escenario más amplio y favorecedor para la penetración de productos chinos, lo que podría "revirar" la ventaja relativa que siempre ha tenido México frente a China en exportaciones de manufactura hacia Estados Unidos.

El que México haya superado a China en el período tratado se debe a varios factores, entre ellos, el que México tenga la "ventaja geográfica" de ser vecino de Estados Unidos -que, entre otros, aminora los costos de transporte- así

Cuadro 3

Estados Unidos de Norteamérica: Importaciones de manufactura con América del Norte y la República Popular China antes y después del TLCAN, (estructura porcentual, 1985-2000)

\begin{tabular}{lrrrrrrrrrrrrrrr}
\hline & 1985 & 1986 & 1987 & 1988 & 1989 & 1990 & 1991 & 1992 & 1993 & 1994 & 1995 & 1997 & 1998 & 1999 & $2000^{*}$ \\
Canadá & 19.2 & 17.74 & 16.86 & 17.71 & 18.2 & 18.14 & 18.4 & 18.33 & 17.86 & 20.98 & 22.4 & 26.54 & 27.29 & 33.56 & 33.24 \\
México & 3.77 & 4.54 & 4.84 & 5.12 & 5.59 & 5.96 & 6.26 & 6.49 & 6.41 & 8.52 & 10.9 & 12.98 & 13.57 & 14.26 & 14.99 \\
& & & & & & & & & & & & & & & \\
RP China & 1.17 & 1.35 & 1.63 & 2.01 & 2.62 & 3.15 & 3.99 & 4.96 & 5.1 & 4.61 & 4.77 & 5.01 & 4.82 & 4.91 & 4.13 \\
\hline
\end{tabular}

Fuente: Estadísticas de 1985 a 1993 de RAMíREZ Bonilla Juan José, México en el área norteamericana de libre comercio, puntos neurálgicos para los países del Pacífico asiático, Colegio de México, 1995 p.102

Fuente: Estadísticas de 1994 -2000 del Centro Internacional de Comercio, www.intracen.org.

Aclaración: El cuadro muestra los porcentajes que representan la importación de manufactura por país de origen del total de importaciones de manufactura de Estados Unidos, en el ámbito global.

*Estimado 
Cuadro 4

Estados Unidos de Norteamérica: importaciones de manufactura con América del Norte y la República Popular China en los primeros meses del 2002. (porcentaje)

\begin{tabular}{lrrr}
\hline & Enero & Febrero & \multicolumn{1}{c}{ Marzo } \\
\hline Canadá & 12.5 & 13.24 & 11.52 \\
México & 18.45 & 23.65 & 32.58 \\
RP China & 25.68 & 36.58 & 26.35 \\
\hline
\end{tabular}

Fuente: Estadísticas del Centro Internacional de Comercio, www.intracen.org. Aclaración: El cuadro muestra los porcentajes que representan la importación de manufactura por país de origen del total de importaciones de manufactura de Estados Unidos, en el ámbito global.

mismo el que durante todos estos años no se hayan acabado completamente las fricciones comerciales entre Estados Unidos y China, así mismo, los beneficios para México como miembro del TLCAN, que le permitieron tener una ventaja aún más significativa.

Como se aprecia en el cuadro 4, de las exportaciones totales de mercancías de México y China en el primer trimestre del 2002, las destinadas a Estados Unidos han tenido una participación más significante en China que en México, excepto en marzo de este año; sin embargo, hacer una evaluación resulta aún muy apresurado, habría que esperar las cifras anuales, la tendencia podría virar.

De acuerdo a las cifras del cuadro 5 podemos identificar que el sector textil manufacturero presenta la mayor importación que hace Estados Unidos de ambos países, con un alto porcentaje respecto del total de productos

\section{Conclusiones}

textiles manufacturados que éste país importa. Así mismo, el sector de metales y otras manufacturas básicas es en el que México tiene más amplia ventaja, en segundo lugar, en el nivel de ventaja, se encuentra el sector textil.

1) Medir las consecuencias del Acuerdo bilateral EUA-China de 1999. 2) Advertir las desventajas para México ante la entrada de China a la OMC.

El comercio bilateral de China y Estados Unidos ha aumentado extremadamente rápido desde la apertura comercial de este país asiático. El flujo de mercancías entre los dos países -la suma de las exportaciones e importacionesaumentó de 1 billón ${ }^{10}$ de dólares en 1978, a 116 billones de dólares en el $2000 .^{11}$

Sin embargo, desde 1993 se presenta una serie de déficit comerciales de Estados Unidos con China, empezando a acentuarse en 1994 con 15.2 billones de dólares. Luego, en 1998, se pensaba era el punto máximo cuando alcanzó los 35.4 billones de dólares, ${ }^{12}$ sin embargo, en el 2000 dicho déficit fue de 84 billones de dólares,

Cuadro 5

Comparación de exportaciones de manufactura de México y China hacia EUA por grupo de producto. (Estimado, 1994-2001)

Parte del total de

Parte del total de

\begin{tabular}{|c|c|c|c|}
\hline Tipo de producto & $\begin{array}{c}\text { Importaciones totales de EUA } \\
\text { de ese tipo de producto }\end{array}$ & $\begin{array}{c}\text { Parte del total de } \\
\text { exportaciones proveniente de } \\
\text { México }\end{array}$ & $\begin{array}{c}\text { Parte del total de } \\
\text { exportaciones proveniente } \\
\text { de China } \\
\end{array}$ \\
\hline Textiles (principalmente prendas de vestir) & $35 \%$ & $23 \%$ & $12 \%$ \\
\hline Equipos y componentes electrónicos & $27 \%$ & $18.10 \%$ & $8.90 \%$ \\
\hline Maquinaria no eléctrica & $18 \%$ & $8.38 \%$ & $9.62 \%$ \\
\hline Metal y otras & & & \\
\hline manufacturas básicas & $29 \%$ & $20.46 \%$ & $8.54 \%$ \\
\hline Calzado & $22 \%$ & $12.13 \%$ & $9.87 \%$ \\
\hline Productos agro-básicos procesados & $26 \%$ & $14 \%$ & $12 \%$ \\
\hline
\end{tabular}


pese al aumento del flujo comercial entre ambos países.

Con el acuerdo bilateral firmado el 15 de noviembre 1999 para la postulación de China como miembro de la OMC, ${ }^{13}$ Estados Unidos pretende reducir el déficit comercial con aquél país. A las empresas estadounidenses se les permitirá comerciar, distribuir y vender bienes, así como auxiliar en la prestación de servicios como mensajería aérea y terrestre, paquetería, almacenaje y publicidad, una vez que China ingrese a la OMC. En el sector de servicios es donde este país realiza una mayor apertura y donde Estados Unidos pretende reducir más su déficit. ${ }^{14}$

China se adhiere a la OMC formalmente en noviembre del 2001; a partir de entonces se fortalece sin duda su posición en la economía global y ello ha suscitado numerosos estudios sobre el impacto económico que esto representará sobre todo para las economías con un similar grado de desarrollo.

En el plano comercial, el propósito debe ser entonces cómo se comportarán las manufacturas chinas en el mercado mundial, ya que representan el "arma" principal con el que China está entrando al nuevo escenario comercial (la OMC) y sobre todo las exportaciones de manufactura hacia el mercado estadounidense, pues tomando en cuenta el nuevo y más importante papel que Estados Unidos juega en el comercio exterior de China serán de importancia central las nuevas relaciones comerciales que se den entre ambos después de la adhesión a la OMC.

Lo que realmente interesa son los efectos de este evento para México; aunque aún es muy temprano analizar estadísticas debemos tomar en cuenta que el TLCAN aún no ha terminado de entrar en vigor, el proceso de desgravación termina en el 2010, y China está ya dentro de la OMC obteniendo beneficios comerciales que permiten la penetración de sus productos a más mercados, sobre todo a Estados Unidos; es de suponer que habrá una amenaza en las exportaciones manufactureras mexicanas en las que aún no termina el proceso de desgravación. ${ }^{15}$ México con urgencia tiene que proponer medidas de protección comercial a sus exportaciones de manufactura, sobre todo las que están presentes en el TLCAN, para evitar consecuencias negativas. ${ }^{16}$ La principal preocupación debe ser ahora procurar una nueva especialización ante el nuevo contexto económico y comercial internacional, ${ }^{17}$ ya que nuestro país debe advertir posibles amenazas no sólo en el mercado externo - ya que China podría representar un competidor manufacturero a nivel internacional más fuerte de lo que ha sido-sino también en el sector interno, principalmente por la falta de especialización de los sectores competitivos.

\section{Notas}

1 Estadísticas del Banco de México para el año 2000.

2 HUERTA Glez. Arturo, 1994 Riesgos del Modelo Neoliberal Mexicano, Diana p.18

3 En noviembre del 2001, China es aceptada como miembro y país subdesarrollado en la Organización Mundial del Comercio; en diciembre de ese mismo año se formaliza su entrada al organismo.

4 López Villafañe Víctor ,1999 Asia en transición. Auge, crisis y desafios, Siglo XXI p.159

5 En las estadísticas publicadas por la Organización Mundial del Comercio para 1999, se muestra en la P. 117, que Estados Unidos ocupa el primer lugar como comprador de manufactura a nivel mundial con un total de 824, 080 millones de dólares, en segundo lugar está Alemania con un total de 326, 060 millones de dólares. Como se puede apreciar la diferencia es abismal.

6 En 1951 Estados Unidos, suspende el Status de Nación más Favorecida (NMF) a todos los países del bloque socialista. En 1952, rompen relaciones diplomáticas y comerciales entre ambos países. Luego, en 1972 con el comunicado de Shangai, las reanudan y se establecen las comerciales con restricciones. En 1979, Estados Unidos otorga una relación comercial "normal" (el status NMF) que se consolida mediante la extensión de la Ley comercial de 1974, la llamada "Extensión Jackson Vanik", ésta otorga a China el Status NMF por parte de Estados Unidos, sin embargo, esta relación comercial estaría sujeta a revisión trianual y a una renovación por parte del Presidente estadounidense, esto si el balance comercial era satisfactorio y se respetaba la calidad de una posible suspensión anual de acuerdo a la consideración del Presidente. Como no existieron déficit comerciales importantes de Estados Unidos con China sino hasta 1993, el Congreso estadounidense empieza a demandar a China las irregularidades en cuestiones laborales, derechos humanos, propiedad intelectual, marcas y patentes; sin embargo, el status de NMF se siguió renovando. Con la renovación del status en 1997, se separan dichos 
asuntos de la renovación.

La relaciones político-comerciales de Estados Unidos, a manera de resumen fueron obtenidas de González Juan, participación en la revista México y la Cuenca del Pacífico vol. 3 núm.9 p. 53 DEP, U de G.

7 El status de Nación Más Favorecida o NMF es una política comercial del Congreso estadounidense que data de principios del siglo $\mathrm{XX}$, el cual otorga una relación comercial "normal" a los países, principalmente subdesarrollados, que pretendan el intercambio comercial con Estados Unidos.

8 El Acuerdo Bilateral firmado en 1997 por Estados Unidos y China sería la última fase de la llamada Extensión Jackson Vanik de 1979. Después del Acuerdo, el status NMF a China ya no estará sujeto a renovación cada tres años como se venía dando desde 1979. China tendrá un más amplio acceso de sus productos al mercado estadounidense.

9 En vísperas de la entrada en vigor de dicho tratado y gracias al proceso de liberalización unilateral, la economía mexicana había logrado dos profundos cambios en sus exportaciones: por un lado tener un mayor acceso a su principal mercado externo Estados Unidos, y por el otro, sus exportaciones se concentraron en los productos manufacturados. Ramírez Bonilla Juan José,1994 México en el área norteamericana de Libre comercio: puntos neurálgicos para los países del pacífico asiático. Colegio de México p.99
10 Tomando en cuenta que un billón de dólares estadounidenses, son mil millones de dólares traducidos a la unidad mexicana.

11 Lardy Nicholas, 2001 "Issues in China's WTO Accession" U.S.-China security Review commission www.brook.edu/foreignpolicystudies.

12 Véase González Juan 2000 "EUA- China : Acuerdo bilateral para el acceso de China a la OMC" p.53 Departamento de Estudios del Pacífico. México y la Cuenca de Pacífico núm. 9.

13 China fue país parte-contratante (junto con otros 23 países) cuando el GATT fue creado en 1947 y su separación formal quedó inconclusa cuando en 1948 decidió unilateralmente, retirarse del mismo. En 1986 hace una nueva solicitud formal de ingreso, lo que consigue en noviembre del 2001.

14 Véase González Juan 2000 México y la Cuenca de Pacífico......

15 Un ejemplo claro son algunos apartados de la industria automotriz.

16 Globermman y Walker, compiladores $1999 E L T L C$, Un enfoque trinacional Fondo de Cultura Económica, p. 35

17 Regalado Raquel (1999) México, ¿sectores competitivos? Siglo XXI p.153 T:? 